\title{
Magnetism and charge order in the ladder compound $\mathrm{Co}_{3} \mathrm{O}_{2} \mathrm{BO}_{3}$
}

\author{
D. C. Freitas, ${ }^{1}$ C. P. C. Medrano, ${ }^{2}$ D. R. Sanchez, ${ }^{2}$ M. Nuñez Regueiro, ${ }^{3}$ J. A. Rodríguez-Velamazán,, 4 \\ and M. A. Continentino ${ }^{1, *}$ \\ ${ }^{1}$ Centro Brasileiro de Pesquisas Físicas, Rua Dr. Xavier Sigaud 150, Urca, 22290-180 Rio de Janeiro, RJ, Brazil \\ ${ }^{2}$ Instituto de Física, Universidade Federal Fluminense, Campus da Praia Vermelha, 24210-346 Niterói, RJ, Brazil \\ ${ }^{3}$ Institut NEEL/CNRS-UJF, Department MCBT, F-38042 Grenoble, France \\ ${ }^{4}$ Instituto de Ciencia de Materiales de Aragon, CSIC Universidad de Zaragoza, E-50009 Zaragoza, Spain \\ ${ }^{5}$ Institut Laue Langevin, CNRS, F-38042 Grenoble, France
}

(Received 25 May 2016; published 4 November 2016)

\begin{abstract}
There are two known ludwigites containing a single transition metal element, $\mathrm{Fe}_{3} \mathrm{O}_{2} \mathrm{BO}_{3}$ and $\mathrm{Co}_{3} \mathrm{O}_{2} \mathrm{BO}_{3}$. The structure of these materials has low-dimensional units in the form of three-legged ladders (3LL) that confer to each of them unique magnetic and electronic properties. $\mathrm{Fe}_{3} \mathrm{O}_{2} \mathrm{BO}_{3}$ presents a staggered charge density wave $(\mathrm{CDW})$ transition in the ladders near room temperature and two magnetic transitions. It has remained a mystery why the other compound $\mathrm{Co}_{3} \mathrm{O}_{2} \mathrm{BO}_{3}$ behaves so conventionally, with a single magnetic transition and no CDW in spite of similar structural and electronic configurations. Neutron diffraction results presented here in this system finally unravel these differences. Far from a trivial explanation, we uncover a coexistence of low and high spin Co ions in well-defined octahedral sites. Our results allow one to solve the contrasting behavior of the Fe and Co ludwigites in terms of a subtle and unique charge ordering mechanism occurring at the microscopic level of the rungs of the $3 \mathrm{LL}$.
\end{abstract}

DOI: 10.1103/PhysRevB.94.174409

\section{INTRODUCTION}

The only two known homometallic oxyborates with a ludwigite structure, $\mathrm{Fe}_{3} \mathrm{O}_{2} \mathrm{BO}_{3}$ and $\mathrm{Co}_{3} \mathrm{O}_{2} \mathrm{BO}_{3}$, have transition metal ions with a local moment and exchange interactions expected to be similar. However, the magnetic, structural, and transport properties of these materials are very different. Understanding the reason for these differences in systems with the same low-dimensional characteristics can lead to deep insight into the nature of magnetism and charge ordering in transition metal compounds. In this paper, we present the solution for why Co behaves so differently from $\mathrm{Fe}$ in the ludwigite structure. We obtain neutron scattering results that yield the magnetic structure of $\mathrm{Co}_{3} \mathrm{O}_{2} \mathrm{BO}_{3}$ and show this is different from that of $\mathrm{Fe}_{3} \mathrm{O}_{2} \mathrm{BO}_{3}$. This is due to a subtle competition between magnetism and charge ordering at the microscopic level of the rungs of the three-legged ladders (3LL) common to both systems. The unique balance attained in each system determines their distinct macroscopic physical properties. In $\mathrm{Co}_{3} \mathrm{O}_{2} \mathrm{BO}_{3}$ nature exploits the low spin of a $\mathrm{Co}^{3+}$ ion to avoid the structural distortion associated with the charge density wave (CDW) formation in $\mathrm{Fe}_{3} \mathrm{O}_{2} \mathrm{BO}_{3}$.

The ludwigites belong to the family of oxyborates. Their structure is formed by subunits consisting of three-legged ladders that confer to them a low-dimensional character (Fig. 2). The oxyborates are known by their complex magnetic behavior [1]. Among these, we mention the partial magnetic ordering in the ludwigites $\mathrm{Fe}_{3} \mathrm{O}_{2} \mathrm{BO}_{3}$ [2,3] and $\mathrm{CoFe}_{2} \mathrm{O}_{2} \mathrm{BO}_{3}$ [4], the random singlet phase in the warwickite $\mathrm{MgTiOBO}_{3}$ [5], and the two-dimensional antiferromagnetism in the hulsite $\mathrm{Co}_{5.52} \mathrm{Sb}_{0.48}\left(\mathrm{O}_{2} \mathrm{BO}_{3}\right)_{2}$ [6].

The ludwigites crystallize in the space group Pbam and their structure can be described by two different types of 3LL. The metallic ions on the ladders occupy the centers of

*mucio@cbpf.br oxygen octahedra. The general formula of these systems is $M_{2}^{2+} M^{\prime 3+} \mathrm{O}_{2} \mathrm{BO}_{3}$, where $M$ and $M^{\prime}$ are divalent and trivalent metallic ions, respectively. There are two known homometallic $\left(M=M^{\prime}\right)$ ludwigites: $\mathrm{Co}_{3} \mathrm{O}_{2} \mathrm{BO}_{3}$ [7] and $\mathrm{Fe}_{3} \mathrm{O}_{2} \mathrm{BO}_{3}[2,3]$. The former orders magnetically near $42 \mathrm{~K}$ and the latter has two ordering temperatures where the magnetic ions in different ladders order at two distinct temperatures, namely, 110 and $70 \mathrm{~K}$. The physical properties of these mixed-valence homometallic ludwigites are quite different. Besides the partial magnetic ordering, $\mathrm{Fe}_{3} \mathrm{O}_{2} \mathrm{BO}_{3}$ [8,9] has a transverse charge density transition near room temperature that has not been found in $\mathrm{Co}_{3} \mathrm{O}_{2} \mathrm{BO}_{3}$. The magnetization curve of $\mathrm{Co}_{3} \mathrm{O}_{2} \mathrm{BO}_{3}$ at the lowest temperature $(T=2 \mathrm{~K})$ is at least one order of magnitude larger than that of the Fe system [7]. This has been taken as an indication that ferromagnetic interactions are more important in the former system. Using neutron powder diffraction (NPD), Bordet et al. [9] have determined the magnetic structure of $\mathrm{Fe}_{3} \mathrm{O}_{2} \mathrm{BO}_{3}$. They have shown that there is a very weak magnetic coupling between the 3-1-3 and 4-2-4 ladders (see Fig. 2) that order independently in this system.

In this paper, we present a neutron powder diffraction study of $\mathrm{Co}_{3} \mathrm{O}_{2} \mathrm{BO}_{3}$, above and below its single magnetic ordering temperature. Our analysis of the neutron data allows us to determine the magnetic structure of this material and reveals some unexpected and interesting behavior. We find a coexistence of low and high spin states Co ions occupying well-defined octahedral sites in the structure. We show that the low-temperature magnetic order implies a charge ordering phenomenon in the system. Our results represent another example of the rich variety of physical behavior exhibited by low-dimensional transition metal oxides.

\section{EXPERIMENT}

The crystals were synthesized according to Freitas et al. [7]. A NPD experiment was carried out in the D1B instrument of 
the Institut Laue-Langevin (ILL) in Grenoble. For the measurements, $3 \mathrm{~g}$ of well-ground needle-shaped black crystals were confined into a cylindrical vanadium can and put inside a cryostat. A wavelength of $1.28 \AA$ was used, which corresponds, in the D1B instrument, to the best compromise between flux, resolution, and absorption of boron. The sample was cooled down to $2 \mathrm{~K}$ and diffraction patterns covering the angular range $0.8^{\circ}-128.8^{\circ}$ were collected for $3 \mathrm{~h}$ at $2,30,50$, and $300 \mathrm{~K}$. The data were analyzed using the FULLPROF Suite programs [10]. The treatments included a full structural refinement of the crystal structure, with a single isotropic atomic displacement parameter (ADP) for each atom type.

\section{RESULTS}

The neutron diffraction patterns show the occurrence of long-range magnetic order with the appearance of a set of magnetic peaks below $T_{N}=42 \mathrm{~K}$, accompanied by a small decrease of background intensity at low angles, as shown in Fig. 1. The magnetic ordering temperature is consistent with previous magnetic measurements [7]. We did not observe structural transitions in the entire range of measured temperatures with the changes of the magnetic structure between 2 and $30 \mathrm{~K}$ accounting for all variations in the spectra. The results of the magnetic structure refinements at $2 \mathrm{~K}$ are given in Table I and the plot of the Rietveld refinement in Fig. 1. The NPD data at $2 \mathrm{~K}$ give lattice parameters of $a=9.317(3)$ $\AA, b=11.950(4) \AA$, and $c=2.9646(7) \AA\left(R_{\mathrm{Bragg}}=8.0 \%\right.$, $\left.R_{F}=6.2 \%\right)$. All magnetic contributions can be indexed with the nuclear unit cell, i.e., with a magnetic propagation vector $\mathbf{k}=\mathbf{0}$. The magnetic structure determination was done by a symmetry analysis, following the representation analysis technique described by Bertaut [11] using BASIREPS software [10]. For the Pbam space group, we found eight real irreducible

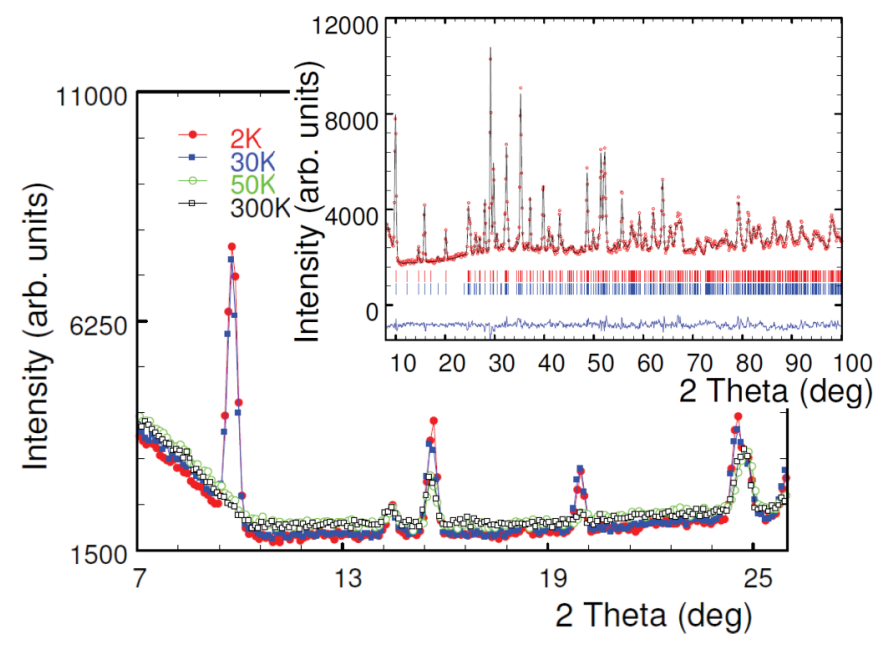

FIG. 1. Low angle part of the neutron diffractograms for $\mathrm{Co}_{3} \mathrm{O}_{2} \mathrm{BO}_{3}$ at $300,50,30$, and $2 \mathrm{~K}$. A set of magnetic peaks is clearly visible below $42 \mathrm{~K}$, as well as magnetic diffuse scattering at higher temperatures. Inset: Rietveld refinements (black line) of the NPD data (red dots) at $2 \mathrm{~K}$. The difference between the experimental data and the Rietveld model is represented by the blue line. Blue (top) and red (bottom) tick marks correspond to the nuclear and magnetic reflection positions, respectively.
TABLE I. Magnetic moments of the ludwigite $\mathrm{Co}_{3} \mathrm{O}_{2} \mathrm{BO}_{3}$ at $2 \mathrm{~K}$ for each direction in $\mu_{B}$ units. $M$ represents the modulus of the magnetic moment vector. $R_{p}=14.5 \%, R_{w p}=13.2 \%, R_{\text {expt. }}=$ $2.05 \%, \chi^{2}=41.4 \%, R_{\text {Bragg }}=8.0 \%, R_{\mathrm{mag}}=8.6 \%$.

\begin{tabular}{lrccc}
\hline \hline Atom (Wyckoff positions) & \multicolumn{1}{c}{$M x$} & $M y$ & $M z$ & $M$ \\
\hline Co4 $(4 h)$ & $-0.5(1)$ & $-0.1(1)$ & 0 & 0.5 \\
Co3 $(4 g)$ & $1.7(1)$ & $3.38(8)$ & 0 & 3.8 \\
Co2 $(2 d)$ & $0.4(2)$ & $3.06(9)$ & 0 & 3.1 \\
Co1 $(2 a)$ & $1.2(1)$ & $-3.4(1)$ & 0 & 3.6 \\
\hline \hline
\end{tabular}

representations for the little group $G k$ associated with $\mathbf{k}=\mathbf{0}$, $\Gamma_{1}-\Gamma_{8}$. Since a single magnetic ordering temperature is observed [7], it is expected that the magnetic structure is described within the same magnetic representation for all magnetic sublattices. Only the odd irreducible representations $\left(\Gamma_{1}, \Gamma_{3}, \Gamma_{5}, \Gamma_{7}\right)$ appear in the magnetic representation $\Gamma_{M}$ for all the Wyckoff positions, with $\Gamma_{5}$ the one that best reproduces the experimental data $\left(R_{\mathrm{mag}}\right.$ values: $\Gamma_{1}=27.2, \Gamma_{3}=25.9$, $\left.\Gamma_{5}=8.6, \Gamma_{7}=13.4\right)$.

The magnetic structure is shown in Fig. 2. The $\mathrm{Co}_{3} \mathrm{O}_{2} \mathrm{BO}_{3}$ system has a ferromagnetic spin configuration in the rungs of the 4-2-4 ladders with an effective moment of $8.2 \mu_{B}$ per cell and a ferrimagnetic configuration in the rungs of the 3-1-3 ladders with about $8 \mu_{B}$ per cell, which gives $1.4 \mu_{B}$ per Co cation. This is in agreement with previous magnetic measurements [7] that found a residual moment of $1.1 \mu_{B} / \mathrm{Co}$ and a predominance of ferromagnetic interactions when compared to $\mathrm{Fe}_{3} \mathrm{O}_{2} \mathrm{BO}_{3}$. All the moments are nearly parallel to the $b$ axis, making this the easy magnetization axis in accord with bulk magnetic anisotropy measurements [12]. Along the $c$ axis the moments in both ladders are ferromagnetically aligned. The magnetic moment in site 2 of the 4-2-4 ladder has a value consistent with a high spin (HS) state of the $\mathrm{Co}^{2+}$

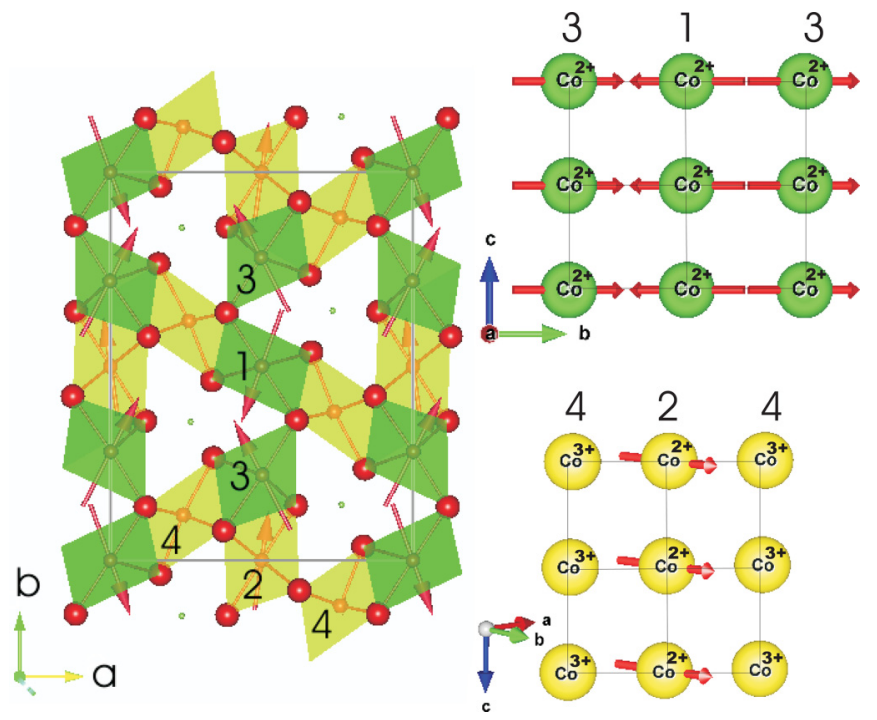

FIG. 2. Magnetic structure proposed for $\mathrm{Co}_{3} \mathrm{O}_{2} \mathrm{BO}_{3}$ by NPD. The two subunits in the form of $3 \mathrm{LL}$ are shown. The numbers indicate the crystallographic sites. Magnetic moments are ferromagnetically aligned along the $c$ axis. 
ion, but the magnetic moment in site 4 is surprisingly small. This suggests that these sites are occupied by $\mathrm{Co}^{3+}$ ions in low spin (LS) states. The sites in the ladder 3-1-3 are all occupied by divalent $\mathrm{Co}^{2+}$ ions in high spin states. They are associated with the large magnetic moments shown in Table I. The value of the magnetic moment expected for $\mathrm{HS} \mathrm{Co}^{2+}$ is $3 \mu_{B}$ and $1 \mu_{B}$ for the LS state, considering only the spin contribution, as is usual for these systems. The values obtained in Table I indicate that there is a reasonable orbital contribution to the moments. Bulk $3 d$ long-range magnetic order in the system due to the interaction between the $3 \mathrm{LL}$ is guaranteed by the presence of a magnetic Co ion on site 2 , which is the one more strongly coupled to the 3-1-3 ladder [9].

\section{DISCUSSION}

The spin state of $\mathrm{Co}^{3+}$ ions in an octahedral environment is determined by a competition between intra-atomic exchange energy (Hund's rule) and crystal field energy [13,14]. The exchange energy favors the high spin (HS) state $\left(t_{2 g}^{4} e_{g}^{2}, S=2\right)$ while the crystal field favors the low spin state (LS) $\left(t_{2 g}^{6} e_{g}^{0}\right.$, $S=0)$. For $\mathrm{Co}^{3+}$ and $\mathrm{Fe}^{2+}$ very often these states are close in energy and include also the possibility of an intermediate spin state (IS) with the configuration $\left(t_{2 g}^{5} e_{g}^{1}, S=1\right)$. Among the Co oxides, $\mathrm{LaCoO}_{3}$ and $\mathrm{GdCoO}_{3}$ exhibit a thermal transition from low spin to high spin, probably with an intervening IS [13-15], and $\mathrm{Co}_{2} \mathrm{O}_{3}$ exhibits a low spin to high spin transition with decreasing pressure [16].

The electron count in a rung of the 4-2-4 ladder corresponds to a background of three $\mathrm{Co}^{3+}$ ions with an extra itinerant electron per rung $[1,8]$. The magnetic configuration obtained by neutrons implies a localization, or charge ordering, of this extra electron on site 2 of the rung, leaving two low spin $\mathrm{Co}^{3+}$ ions in the two outer sites 4 of the rung and a $\mathrm{Co}^{2+}$ on the center. Since site 2 is a symmetric site, this charge ordering is not necessarily accompanied by a structural transition as in the case of the Fe homometallic ludwigite.

The susceptibility of the Co ludwigite above the ordering temperature obeys a Curie-Weiss law [7] that allows one to obtain the effective magnetic moment. This turns out to be $p_{\text {eff }}=7.2 \mu_{B}$, to be compared with the value of $7.4 \mu_{B}$ expected if all the $\mathrm{Co}$ ions including the trivalent ones are in high spin states and there is no orbital contribution. Since these values are very close, the crossover or transition from HS to LS spin could be present in $\mathrm{Co}_{3} \mathrm{O}_{2} \mathrm{BO}_{3}$. Useful information on the spin states can also be obtained from the temperature-dependent entropy at the magnetic transition. Figure 3 shows the entropy curves obtained from the measured specific heats for three ludwigites, the two homometallic [3,7] and for $\mathrm{Co}_{2.5} \mathrm{Sn}_{0.5} \mathrm{O}_{2} \mathrm{BO}_{3}$ [17] that has only $\mathrm{Co}^{2+}$ ions. The $\mathrm{Co}_{3} \mathrm{O}_{2} \mathrm{BO}_{3}$ has the smallest entropy at $T_{N}$, considerably less than the expected value of $36.41 \mathrm{~J} / \mathrm{mol} \mathrm{K}$ if all $\mathrm{Co}$ ions were in the HS states $[S=R \ln (2 S+1)]$. The theoretical value for the entropy released at the magnetic transition for $\mathrm{Co}_{2.5} \mathrm{Sn}_{0.5} \mathrm{O}_{2} \mathrm{BO}_{3}$, where all $\mathrm{Co}$ ions are in $\mathrm{Co}^{2+}$ high spin states, is $28.8 \mathrm{~J} / \mathrm{mol} \mathrm{K}$. For $\mathrm{Co}_{3} \mathrm{O}_{2} \mathrm{BO}_{3}$ assuming $\mathrm{Co}^{2+}$ in $\mathrm{HS}$ and $\mathrm{Co}^{3+}$ in LS states, the expected entropy is $23.0 \mathrm{~J} / \mathrm{mol} \mathrm{K}$, still larger than the experimental value, as can be checked in Fig. 3.

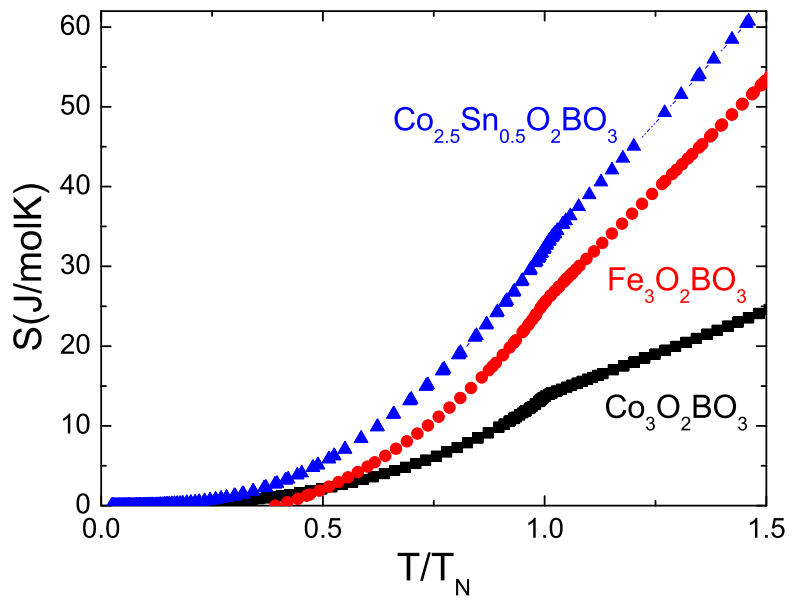

FIG. 3. Entropy curves for the homometallic ludwigites and for $\mathrm{Co}_{2.5} \mathrm{Sn}_{0.5} \mathrm{O}_{2} \mathrm{BO}_{3}$ obtained from specific heat results as functions of temperature $[3,7,17]$. The entropy release at $T_{N}, S\left(T_{N}\right)=$ $13.71 \mathrm{~J} / \mathrm{mol} \mathrm{K}$ for $\mathrm{Co}_{3} \mathrm{O}_{2} \mathrm{BO}_{3}$, is much smaller than the expected value of $36.41 \mathrm{~J} / \mathrm{mol} \mathrm{K}$ if all the Co ions were in HS states.

We now compare the magnetic structures and properties of the two homometallic ludwigites as obtained in the neutron scattering experiments. First, the magnetic ordering in the $\mathrm{Co}$ system is a single step phenomenon in which the magnetic moments in the two different ladders order simultaneously. This is in contrast to $\mathrm{Fe}_{3} \mathrm{O}_{2} \mathrm{BO}_{3}$ where 4-2-4 ladders order at $110 \mathrm{~K}$ and the 3-1-3 ladders order at $70 \mathrm{~K}$. As concerns the magnetic structures, the moments in the ladders are coupled in different ways in these systems. In the Fe system the magnetic moments in a rung of the 4-2-4 ladders couple ferromagnetically, pointing along the $b$ axis [9]. The coupling between the magnetic moments in consecutive rungs of these ladders along the $c$ axis is antiferromagnetic. Notice that due to the staggered CDW ordering at $\approx 283 \mathrm{~K}$ one should distinguish between sites $4 a$ and $4 b$ in the 4-2-4 ladders of $\mathrm{Fe}_{3} \mathrm{O}_{2} \mathrm{BO}_{3}$. However, the moments in these sites were found to be nearly the same [9]. $\mathrm{Co}_{3} \mathrm{O}_{2} \mathrm{BO}_{3}$ has a ferromagnetic spin configuration in the rungs of the 4-2-4, but along the $c$ axis the moments are ferromagnetically aligned. In $\mathrm{Fe}_{3} \mathrm{O}_{2} \mathrm{BO}_{3}$, the magnetic moments in the 3-1-3 ladders have a ferrimagnetic coupling along a rung with the moments pointing into the $a$ direction. These in turn are ferromagnetically coupled along the $c$ axis [9]. The $\mathrm{Co}_{3} \mathrm{O}_{2} \mathrm{BO}_{3}$ has a ferrimagnetic configuration but the moments are all pointing almost into the $b$ direction.

In both homometallic ludwigites, ferromagnetic ordering between the moments in a rung of the 4-2-4 ladders favors delocalization of this extra electron that gains kinetic energy. Since there is no structural transition in $\mathrm{Co}_{3} \mathrm{O}_{2} \mathrm{BO}_{3}$, this delocalized electron could partially Kondo screen the moments in the rung of this ladder. This mechanism, however, is not sufficient to explain the small value of the magnetic moments found in the neutron experiments and especially why this occurs for the moments on sites 4 and not for that on site 2. Also in the oxyborates there are many competing magnetic interactions. The moments at site 4 are connected through different superexchange paths to several other magnetic ions in the structure. This results in conflicting information with 


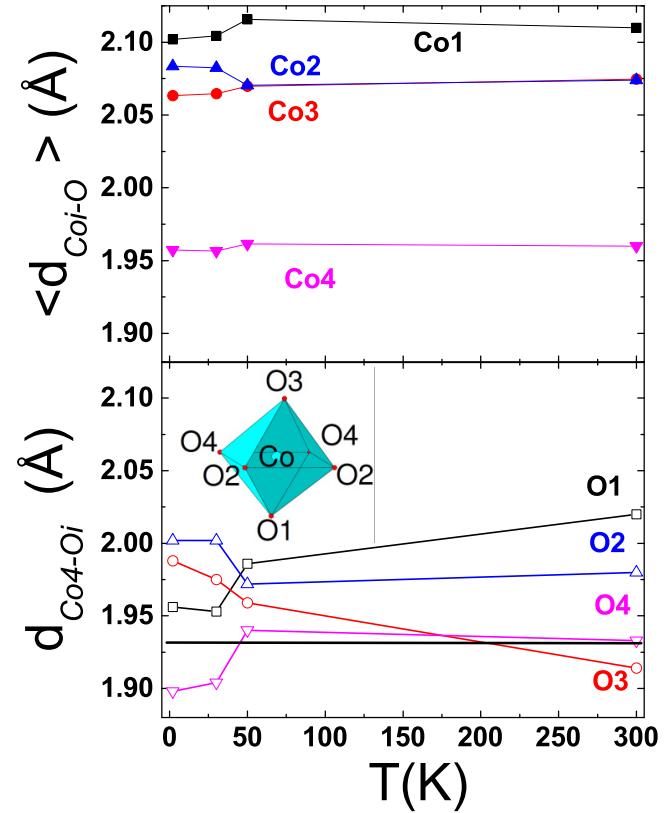

FIG. 4. Upper panel: Weighted average Co-O distances for each of the four Co sites as a function of temperature. Lower panel: $\mathrm{Co}-\mathrm{O}$ distances of the octahedra in site 4 of $\mathrm{Co}_{3} \mathrm{O}_{2} \mathrm{BO}_{3}$ obtained by NPD measurements. The line shows the threshold distance, obtained for a $\mathrm{CoO}_{6}$ cluster by Chen et al. [19], where the HS and LS states exchange stability.

a cost in energy for the system. A nonmagnetic, $\mathrm{LS} \mathrm{Co}^{3+}$ occupying this site avoids the system to have to account for this energy cost.

In an effort to clarify the mechanism responsible for the coexistence of high and low spin states in the Co ludwigite, we calculated the $V_{z z}$ component of the electric field gradient (EFG) on the different Co sites surrounded by oxygen octahedra. This parameter is a measure of the deviation of the environment from symmetric octahedra. Following Ivanova et al. [12] and taking the $z$ axis along the major axis of the oxygen octahedra, we calculated $V_{z z}$ for all the Co sites. We find that site 4 has the smallest $V_{z z}$, showing that this is the most symmetric site in the compound. When lowering the temperature, this parameter decreases, going from $V_{z z}(4)=0.0322 e / \AA^{3}$ at room temperature to $V_{z z}(4)=0.0145 e / \AA^{3}$ at $2 \mathrm{~K}$, indicating that the oxygen octahedra surrounding site 4 become even more symmetric at low temperatures. For comparison, notice that in $\mathrm{LaCoO}_{3}$, an archetypical compound with $\mathrm{Co}^{3+}$ ions in the LS state at low temperatures, $V_{z z}=0.00244 e / \AA^{3}$ at $5 \mathrm{~K}[15,18]$. In a recent study of trivalent Co ions in sites with octahedral symmetry (with $V_{z z}=0.0117 e / \AA^{3}$ ), Chen et al. [19] have shown a close relation between the spin states and the Co-O distances in the octahedra. The intermediate spin (IS) states are always higher in energy, but the HS and LS states exchange stability at a distance of $\sim 1.932 \AA$, the latter becoming more stable at shorter distances. As we can see in Fig. 4, Co-O distances below this limit are observed only for site 4 , yielding in this case a favorable condition for a low spin state. Notice that site 4 is the one with shorter weighted average distances.

\section{CONCLUSIONS}

In summary, neutron powder diffraction as a function of temperature in the compound $\mathrm{Co}_{3} \mathrm{O}_{2} \mathrm{BO}_{3}$ allowed us to obtain its magnetic structure in the ordered phase, which is shown in Fig. 2. The magnetic transition occurs near $42 \mathrm{~K}$, with a ferromagnetic coupling between the $a b$ planes and a ferrimagnetic coupling in this plane. The moments order close to the $b$ direction, in agreement with previous bulk magnetic anisotropy measurements [7,12]. We found that Co ions on sites 4 of the 4-2-4 ladders have a surprisingly small magnetic moment, consistent with low spin $\mathrm{Co}^{3+}$ ions occupying these sites below the ordering temperature. The magnetic moments on the remaining sites of the ladders, including those on sites 2 of the 4-2-4 ladders, are in agreement with these sites being occupied by $\mathrm{Co}^{2+}$ ions in high spin states. This coexistence of low and high spin Co ions has been found previously in oxides [13-16], but this is time it is found in a ludwigite, a system where the metallic ions occupy only octahedral sites. A subtle microscopic mechanism occurs at the level of the rungs of the 4-2-4 3LL in which the itinerant electron shared by the three $\mathrm{Co}^{3+}$ ions on a rung of this ladder localizes on site 2 of the rung. The charge ordering of this itinerant electron leaves two low spin $\mathrm{Co}^{3+}$ ions in the outer sites 4 of this ladder and a magnetic $\mathrm{Co}^{2+}$ with the localized electron on site 2. The low spin state of the ions occupying site 4 is favored by the highly symmetrical octahedral environment of these sites and their short Co-O distances. Furthermore, a nonmagnetic $\mathrm{Co}^{3+}$ on these sites adds positively to the energy balance by avoiding frustration in the system. This unique charge and magnetic balance allows for the Co ludwigite to avoid the structural distortion associated with the staggered CDW observed in the Fe system.

\section{ACKNOWLEDGMENTS}

Support from the Brazilian agencies CNPq and FAPERJ is gratefully acknowledged. We are grateful to Institut LaueLangevin for neutron beam time allocation. J.A.R.-V. acknowledges CSIC for a JAEdoc contract.
[1] M. A. Continentino, J. C. Fernandes, R. B. Guimarães, B. Boechat, and A. Saguia, in Frontiers in Magnetic Materials, edited by A. V. Narlikar (Springer, Berlin, 2005), pp. 385-410.

[2] R. B. Guimarães, M. Mir, J. C. Fernandes, M. A. Continentino, H. A. Borges, G. Cernicchiaro, M. B. Fontes, D. R. S. Candela, and E. Baggio-Saitovich, Phys. Rev. B 60, 6617 (1999).
[3] J. C. Fernandes, R. B. Guimarães, M. A. Continentino, L. Ghivelder, and R. S. Freitas, Phys. Rev. B 61, R850 (2000).

[4] D. C. Freitas, M. A. Continentino, R. B. Guimarães, J. C. Fernandes, E. P. Oliveira, R. E. Santelli, J. Ellena, G. G. Eslava, and L. Ghivelder, Phys. Rev. B 79, 134437 (2009). 
[5] T. G. Rappoport, L. Ghivelder, J. C. Fernandes, R. B. Guimarães, and M. A. Continentino, Phys. Rev. B 75, 054422 (2007).

[6] D. C. Freitas, R. B. Guimarães, J. C. Fernandes, M. A. Continentino, C. B. Pinheiro, J. A. L. C. Resende, G. G. Eslava, and L. Ghivelder, Phys. Rev. B 81, 174403 (2010).

[7] D. C. Freitas, M. A. Continentino, R. B. Guimarães, J. C. Fernandes, J. Ellena, and L. Ghivelder, Phys. Rev. B 77, 184422 (2008).

[8] M. Mir, R. B. Guimarães, J. C. Fernandes, M. A. Continentino, A. C. Doriguetto, Y. P. Mascarenhas, J. Ellena, E. E. Castellano, R. S. Freitas, and L. Ghivelder, Phys. Rev. Lett. 87, 147201 (2001).

[9] P. Bordet and E. Suard, Phys. Rev. B 79, 144408 (2009).

[10] J. Rodriguez-Carvajal, Physica B 192, 55 (1993); FULLPROF Suite, http://www.ill.eu/sites/fullprof/.

[11] E. F. Bertaut, Acta Crystallogr. Sect. A 24, 217 (1968).

[12] N. B. Ivanova et al., J. Exp. Theor. Phys. 113, 1015 (2011); N. V. Kazak, N. B. Ivanova, V. V. Rudenko, S. G. Ovchinnikov, A. D. Vasil'ev, and Yu.V. Knyazev, Solid State Phenom. 152-153, 104 (2009).
[13] P. M. Raccah and J. B. Goodenough, Phys. Rev. 155, 932 (1967); J. Appl. Phys. 39, 1209 (1968); M. A. SenarisRodriguez and J. B. Goodenough, J. Solid State Chem. 116, 224 (1995).

[14] M. A. Korotin, S. Y. Ezhov, I. V. Solovyev, V. I. Anisimov, D. I. Khomskii, and G. A. Sawatzky, Phys. Rev. B 54, 5309 (1996); D. L. Khomskii and U. Löw, ibid. 69, 184401 (2004).

[15] P. G. Radaelli and S.-W. Cheong, Phys. Rev. B 66, 094408 (2002).

[16] R. A. Bari and J. Sivardière, Phys. Rev. B 5, 4466 (1972).

[17] C. P. C. Medrano, D. C. Freitas, D. R. Sanchez, C. B. Pinheiro, G. G. Eslava, L. Ghivelder, and M. A. Continentino, Phys. Rev. B 91, 054402 (2015).

[18] J.-F. Lin et al., Phys. Rev. B 73, 113107 (2006).

[19] J.-M. Chen et al., J. Am. Chem. Soc. 136, 1514 (2014); M. W. Haverkort, Z. Hu, J. C. Cezar, T. Burnus, H. Hartmann, M. Reuther, C. Zobel, T. Lorenz, A. Tanaka, N. B. Brookes, H. H. Hsieh, H.-J. Lin, C. T. Chen, and L. H. Tjeng, Phys. Rev. Lett. 97, 176405 (2006). 